LAWRENCE

LIVERMORE

NATIONAL

LABORATORY

\title{
Comparing Three Types of Numerical Techniques for the Integration of Perturbed Satellite Motion
}

E. Robinson

June 22, 2010 
This document was prepared as an account of work sponsored by an agency of the United States government. Neither the United States government nor Lawrence Livermore National Security, LLC, nor any of their employees makes any warranty, expressed or implied, or assumes any legal liability or responsibility for the accuracy, completeness, or usefulness of any information, apparatus, product, or process disclosed, or represents that its use would not infringe privately owned rights. Reference herein to any specific commercial product, process, or service by trade name, trademark, manufacturer, or otherwise does not necessarily constitute or imply its endorsement, recommendation, or favoring by the United States government or Lawrence Livermore National Security, LLC. The views and opinions of authors expressed herein do not necessarily state or reflect those of the United States government or Lawrence Livermore National Security, LLC, and shall not be used for advertising or product endorsement purposes.

This work performed under the auspices of the U.S. Department of Energy by Lawrence Livermore National Laboratory under Contract DE-AC52-07NA27344. 


\title{
Comparing Three Types of Numerical Techniques for the Integration of Perturbed Satellite Motion
}

\author{
Eric Robinson \\ United States Air Force Academy \\ Department of Mathematics, Mathematics Major \\ c11eric.robinson@usafa.edu
}

\begin{abstract}
This project compares the computational efficiency, stability/accuracy, and precision of various numerical integration schemes to determine which is most appropriate for use in conjunction analysis of satellite orbits. Specifically, this project examines a few of the top Runge-Kutta, extrapolation, and multistep algorithms, combining both previous research in this field with new analysis to form the final conclusion. In addition, a method is proposed for using dense output to expedite orbit calculations.
\end{abstract}

Key Words: numerical integration, Runge-Kutta, extrapolation, multivalue/multistep predictorcorrector methods, dense output

\section{Introduction}

The ultimate goal of considering satellite motion above the earth is to determine where a satellite will be located given a known initial location, initial velocity, and a descriptive model of how the position of that satellite evolves with time. Since these models are based on Newton's Second Law with respect to the motion of the satellite, they usually encompass a system of three ordinary differential equations (one for each component of the standard basis for $\mathbb{R}^{3}$ ), or ODEs, each involving an acceleration term describing the net force acting on a satellite at any time. Though some theoretical models may have analytical closed-form solutions for the position of a satellite at any time (such as the result of Kepler's Two-Body Problem), most applications take into account the effects of other bodies such as the sun, moon, or even how Earth's imperfect spherical shape effects its gravitational pull. These outside effects on the satellite are called perturbations and the resulting motion is described as being perturbed. As should be expected, the vast majority of these models cannot be solved analytically, which means that solutions at various points in time need to be determined via numerical integration techniques.

While some numerical techniques have been developed to directly integrate systems of second-order differential equations, such as many satellite models based on Newton's Second Law, most are designed to work on systems of first-order equations. We will only analyze the methods for first-order systems as the methods designed for second order systems become inefficient for nonconservative systems. Thus, all celestial models will be transformed into a system of six first-order ODEs.

Once this set-up is completed, the question becomes which of the available numerical methods is best suited for the job of integrating satellite orbit ODE models. As previously described, models of perturbed motion can be highly nonlinear and nonautonomous, meaning that a single evaluation of the model (referred to as a "function evaluation") costs much 
computational effort. In addition, for applications involving the integration of perturbed orbits of multiple satellites, any excess computational time can quickly become magnified. However, it is also important to ensure that any methods implemented are both stable (i.e. they tend to converge to the correct solution as step size increases) and meet desired precision/accuracy requirements.

This report will focus on first determining the stability of numerical techniques, and then look at which techniques are best for integrating satellite orbit motion models at various predefined error tolerances, closing with a recommendation about the best method to implement for general satellite use. In order to reach this end, various algorithms from the three main classes of numerical integration techniques-Runge-Kutta, extrapolation, and multivalue/multistep methods - will be evaluated. For background on how these families of numerical methods are constructed see [1], [5], or [8].

\section{Methodology}

The idea of stability in numerical integration of ODEs brings into focus the concept of stiffness in the ODE model. Stiffness occurs when the solution to a model changes on two very different scales. Fortunately, stiffness requires a transient convergence to a stable final solution (see [3] for more about this), which requires either the existence of a limit cycle or attracting fixed point. Since satellite orbits are not isolated and do not converge to a single final trajectory, their models are not stiff. Therefore, we do not need to consider methods that are made exclusively to solve stiff systems. This is very helpful for computational efficiency because algorithms for solving stiff systems require evaluations of the Jacobian matrix, which can be equivalent to multiple function evaluations in computational time, so we know that non-stiff solvers will maximize efficiency at no expense to precision or accuracy.

A great deal of pioneering work was done by O. Montenbruck in [4] on the subject of efficiency of numerical integration techniques for satellite orbits. Montenbruck compared a large variety of many different algorithms on the test case two-body problem. His findings indicated that the most efficient algorithms were the $11^{\text {th }}$ order Runge-Kutta-Nystrom method for both low and high precision/accuracy, followed closely by the variable-order and stepsize predictor-corrector, multivalue/multistep method of Shampine and Gordon (called DE) and the variable- order and stepsize Gragg-Bulirsh-Stoer (GBS) extrapolation method, with the efficiency modifications and stepsize/order control algorithm contained in [1], referred to as ODEX. Montenbruck also mentions the possibility of using dense output algorithms to vastly increase efficiency, but some of these methods were very young and therefore had not yet been sufficiently analyzed. Dense output simply means that we perform some sort of interpolation scheme on each step, allowing us to find output at any number of predefined points. The benefit of dense output is that it allows us to integrate from the initial time to the final time and then use interpolation to find the state vectors at various predefined times. This eliminates the need to stop integration routines prematurely at each point, thereby increasing efficiency. For a discussion of how this works and an analysis about interpolation error we refer the reader to [1].

This study compares the best algorithms from each of the three families of numerical integration techniques - Runge-Kutta, extrapolation, and multivalue/multistep methods - as determined by Montenbruck's study. However, new findings and improved implementations of the various numerical integration techniques have emerged since Montenbruck's study. Therefore, the study implements these methods in their most current versions to see if any of the 
aforementioned new findings have affected the relative results of the premier algorithms in each family.

We determined that the best method to analyze from the Runge-Kutta family was the eight order method RK 8(5,3) presented in [8] which was found by Dormand and Prince due to its seventh order error estimator, allowing for variable stepsize control, existence of a seventh order dense output option for this specific method, and well-documented analysis in [1]. However, it should be noted that recent developments in $9^{\text {th }}$ order Runge-Kutta methods have been found and are presented in [10], [11], and [12] which may offer improved performance. The GBS method was used as the representative from the extrapolation class as it can obtain very high orders due to the even global error series of the modified midpoint method, the results of which are subject to extrapolation. In addition, Hairer and Ostermann present a method in [2] to provide dense output for the GBS method while maintaining its high orders, making it an ideal candidate for comparison (a further explanation is provided in [1]). Finally, the method ode113 in MATLAB developed by Shampine and Reichelt was chosen as the multivalue/multistep algorithm due to its ability to efficiently obtain high orders while also having been programmed by its designer. Details about this method are presented in [9], which show that its predecessor was the same algorithm used in the method DE mentioned in [4] and [5].

The Runge-Kutta-Nystrom Methods, found to be so effective in integrating the two-body problem, were not evaluated by this study. This is because these methods are designed directly integrate systems of second order ODEs, but are only efficient in comparison to the other methods if the model is conservative, i.e. does not depend upon velocity. However, the full force model that is needed for conjunction analysis includes a space drag term, which is dependent upon velocity. In such a case, the information provided by Montenbruck in his study identifies these methods as not being competitive with either GBS or DE/ode113.

In order to begin a comparison of methods, we must first develop a test model that will be used for evaluation. The full force model that will ultimately be used for conjunction analysis also includes many other perturbing terms aside from space drag, to include forces from the sun, moon, and even changes to Earth's gravitational field caused by such events as the movement of waves in the oceans. Such a model is far too complicated to have a closed-form analytical solution. Thus, we cannot use this system for our study as it affords us no method of determining absolute error rather than simple error estimates. However, in order to obtain the proper results, we should create some sort of test model that sufficiently simulates the sporadic trajectories that can be created by the full force model. This means that the system created by the two-body problem, even though it provides a closed-form solution for finding absolute error, should not be used as the test model, for its solutions are much too regular in nature. This kind of oversimplified model could cause an unwanted bias in our analysis, such as predicting that a Runge-Kutta-Nystrom method would be more efficient than another method due to the conservative nature of the two-body problem, when in fact this is an incorrect conclusion.

These considerations led to the creation of the test model described by equations 1 . This system simulates the three essential parts of the full force model: Earth's gravitational field, space drag, and perturbations caused by the sun, moon, and other objects. 
(1)

$$
\begin{gathered}
\dot{x}=v_{x} \\
\dot{y}=v_{y} \\
\dot{z}=v_{z} \\
\dot{v}_{x}=-D v_{x}-\frac{K x}{R^{\frac{3}{2}}}+5 \sin \left(\frac{t}{1000 s}\right) \frac{m}{s^{2}} \\
\dot{v}_{y}=-D v_{y}-\frac{K y}{R^{\frac{3}{2}}}+5 \cos \left(\frac{t}{1000 s}\right) \frac{m}{s^{2}} \\
\dot{v}_{z}=-D v_{z}-\frac{K z}{R^{\frac{3}{2}}}+5 \sin \left(\frac{t}{1000 s}\right) \frac{m}{s^{2}} \\
K=G M_{E} \approx 3.986004415 \times 10^{14} \frac{m^{3}}{s^{2}}, D=5 \times 10^{-8} s^{-1}, R=6.65256 \times 10^{6} \mathrm{~m}
\end{gathered}
$$

In this model, $x, y$, and $z$ are the coordinates of the satellite relative to the center of the earth, $\dot{v}_{x}$, $\dot{v}_{y}$, and $\dot{v}_{z}$ are the satellites components of velocity, $G$ is the gravitational constant, $M_{E}$ is the mass of the earth, $D$ is the coefficient of space drag, and $R$ is the distance from the center of the center of the earth. The first term in each of the acceleration equations simulates space drag, the second term simulates Earth's gravitational force on the satellite, and the third term simulates the sum of all other perturbations at some time $t$. The closed-form solution to this system is shown in Appendix A.

This model was designed with two major assumptions that allowed for it to have a closed-form solution. First of these was that the center of the earth always remains at the origin. This is a valid assumption as the masses of satellites orbiting the earth can be considered as negligible compared to the mass of the earth. In addition, movements of the earth caused by its gravitational attraction to the moon will simultaneously move the satellite, so this effect is lumped into the perturbation category instead of being simulated by the three body problem. The second was that the initial speed $v_{0}$ of such a satellite should be similar to the speed of an object orbiting in uniform circular motion at a distance $R$ from the center of Earth, which is calculated in equations 2 .

(2)

$$
v_{0}=\sqrt{\frac{4 \pi^{2} R^{2}}{(5400 s)^{2}}} \approx 7740.6 \frac{\mathrm{m}}{\mathrm{s}}
$$

$$
\left[\begin{array}{c}
x(0) \\
y(0) \\
z(0) \\
v_{x}(0) \\
v_{y}(0) \\
v_{z}(0)
\end{array}\right]=\left[\begin{array}{c}
R \\
0 \\
0 \\
0 \\
v_{0} \\
0
\end{array}\right]=\left[\begin{array}{c}
6.65256 \times 10^{6} \mathrm{~m} \\
0 \\
0 \\
0 \\
7740.6 \frac{\mathrm{m}}{\mathrm{s}} \\
0
\end{array}\right]
$$

The result of this calculation led to the creation of the initial state vector in (3) at time zero. For the remainder of this study, each integration will begin from this initial state unless otherwise stated. Also, whenever we refer to the "initial vector" we mean this vector.

Using this test model, the algorithms will be compared in the following manner. To analyze stability, each method will be integrated across a set time change at various error 
tolerances for two slightly different initial trajectories. The difference will then be compared to what the true analytical difference should have been to see which method is the most stable. Then, the algorithms will be run with varying error tolerance levels across a set time change, comparing computational time to error tolerance and absolute error. Finally, each algorithm will be used to find a solution across a set of predefined points, which will be compared to the solution found by dense output at those same points.

\section{Code Design of Algorithms}

All of the methods compared by this study were implemented using MATLAB. Since the multistep method ode113 comes built-in with MATLAB with efficiency statistics, this code was run without modification. The other two methods, RK $8(5,3)$ and GBS, were programmed using information as reference material from [1], [5], and [8].

In addition to vectorizing the MATLAB code for increased efficiency, two main issues were considered in creating RK $8(5,3)$ and GBS programs. The first was that we wanted our initial stepsize to be as close to optimal as possible. This is important because a bad initial stepsize estimate could lead to a series of rejected steps, which would be very costly. In order to create an estimate that would be close to the initial stepsize without being in danger of rejection, a routine was created that found the approximate acceptable initial stepsize for the two algorithms at various levels of error tolerance. The results were plotted in figure 1. Error tolerance is measured in meters.
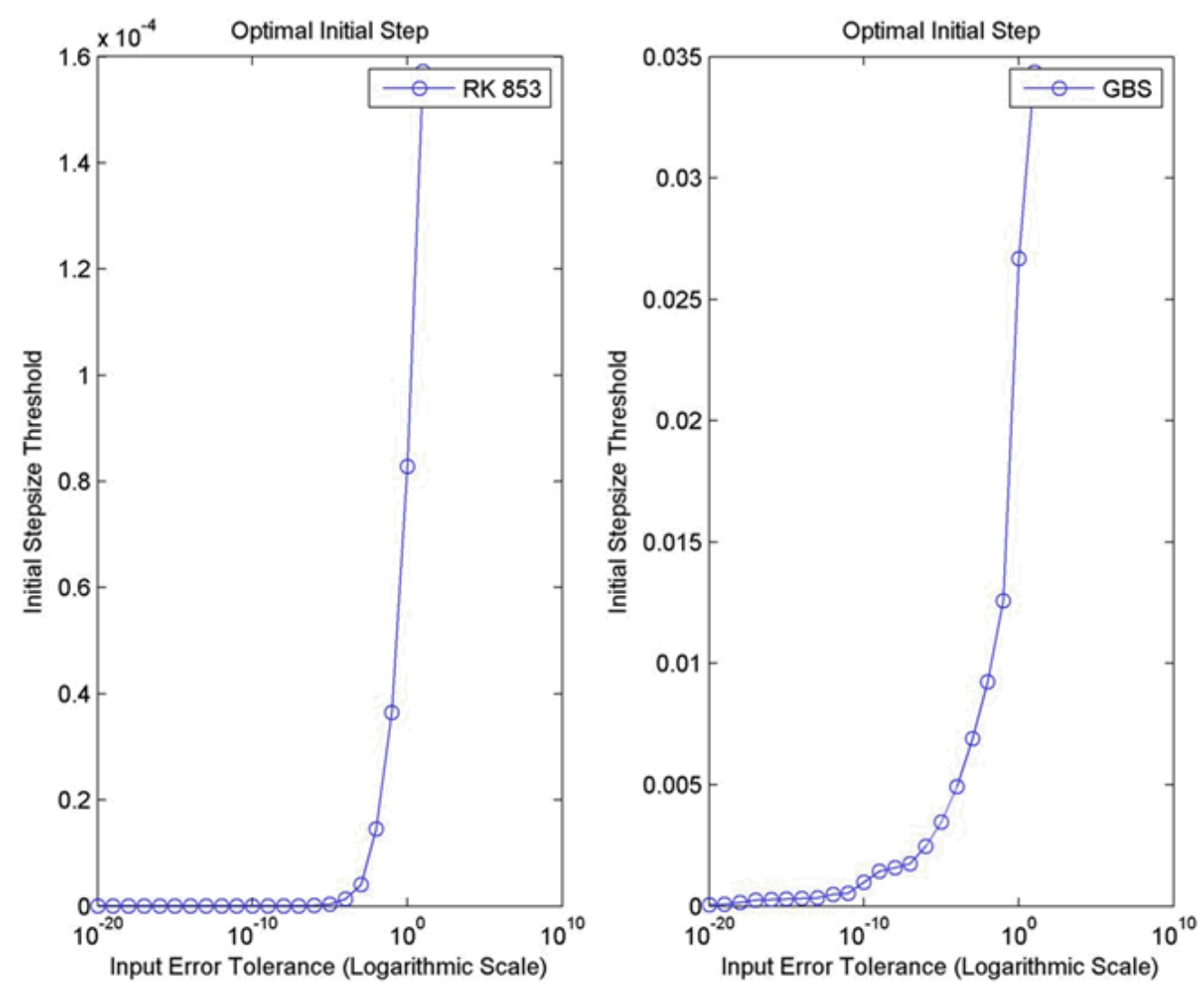

Figure 1-Threshold for optimal initial steps 
From Taylor's Theorem, we know that error varies proportionally to some power of power of stepsize, i.e. error $\approx a(h)^{b}$ for some constants $a$ and $b$, where $h$ is the stepsize. Thus, we expect that the curves shown in figure 1 should be estimated with a power model where Step $=$ $c(h)^{d}$ for some constants $c$ and $d$. Using the regression analysis tools in MATLAB's curve fitting tool, the best fit curve of this form for RK 8(5,3) was $h=5.301 \times 10^{-5}$ (Error) 0.5024 and for GBS was $h=.0234$ (Error) ${ }^{0.1772}$. The $r^{2}$ values for these fitted curves were 0.9457 for RK $8(5,3)$ and 0.9857 for GBS. Thus, these models were good fits. However, in order to insure that the initial step is almost never rejected, we multiplied each model by a factor of 0.9 .

The second issue considered in creating these programs was the value of the factor of safety $S$ used in the stepsize control algorithm. This only pertained to the RK $8(5,3)$ algorithm as the GBS algorithm was completely defined by the method outlined in [1] and repeated in [8]. In the RK $8(5,3)$ program, the stepsize control algorithm scales the current step by a factor that is meant to keep the error at the desired level while maximizing computational efficiency. Since RK $8(5,3)$ has a seventh-order error estimator according to [8], it scales as $O\left(h^{8}\right)$ where $h$ is the stepsize. Thus, after the $k^{\text {th }}$ iteration, we have

$$
h_{k+1} \approx h_{k}\left|\frac{e r r_{k+1}}{e r r_{k}}\right|^{\frac{1}{8}}
$$

where $\operatorname{err}_{k}$ is the magnitude of the error at the $k^{\text {th }}$ step and $e r r_{k+1}$ is the magnitude of the desired error at the next step. However, this is only an estimate, so in order to minimize the number of costly rejected steps, we build a factor of safety $S$ with $0<S<1$ into the actual stepsize control algorithm so that

$$
h_{k+1}=S h_{k}\left|\frac{e r r_{k+1}}{e r r_{k}}\right|^{\frac{1}{8}} \text {. }
$$

After a few initial test runs of RK $8(5,3)$ as compared to GBS and ode113, it seemed that RK $8(5,3)$ began to become very inefficient around an absolute error tolerance of 0.0001 . Thus, we endeavored to further the longevity of the algorithm by adapting the factor of safety to make RK $8(5,3)$ as efficient as possible around this absolute error tolerance. To do this, we ran two test integrations, across 10 seconds and then 20 seconds, to see where the most efficient factor of safety occurred (this is long enough for the stepsize to settle into its natural rhythm so we will obtain a good estimate for the factor of safety). The results are plotted in figure 2 . From these graphs, we can see that the ideal factor of safety was $S=0.8$, so this was chosen for integration.
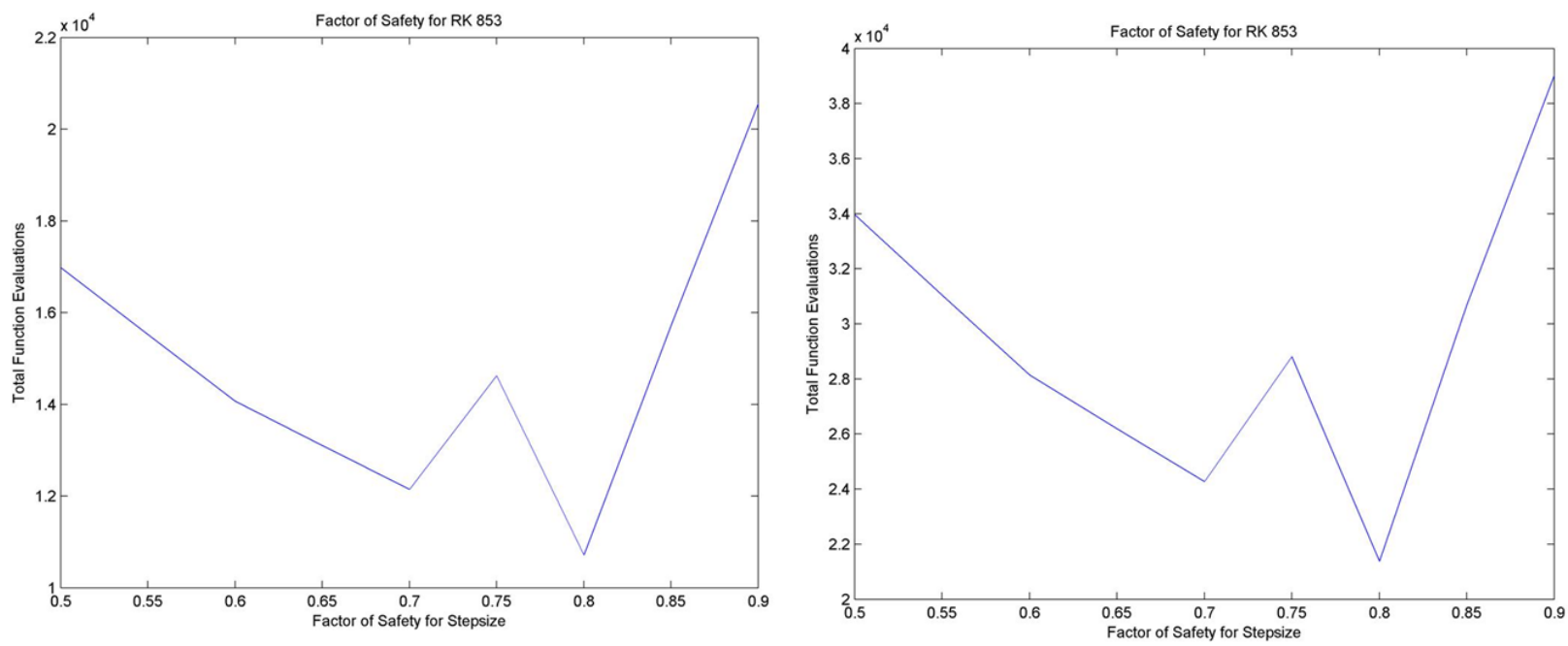

Figure 2-Factor of safety in RK $\mathbf{8}(5,3)$ stepsize control algorithm 
The remainder of the routines for RK $8(5,3)$ and GBS were written completely from the descriptions found in [1], [5], and [8]. The only other notable piece of information was that the stepsize control algorithms utilized local error estimators instead of global. This was because the existing algorithms are much more adapted to this method of stepsize control than using global error. Thus, when actually implementing these algorithms, if a global absolute and relative tolerance is desired, then we can either divide our local error tolerances by a scaling factor, or we can implement a revision in our stepsize control algorithm (one such revision is presented on pages 913-914 of [8]). If the former alternative is chosen, the analysis of this study suggests that dividing the error tolerance by the dimensionless scaling factor $\left(10 \mathrm{~s}^{-1}\right)\left(t_{f}\right)$ where $t_{f}$ is the final time in seconds of the integration, should be sufficient. If this is not the case, then we simply increase the coefficient of $t_{f}$ until the global error estimation is acceptable and then record this new coefficient for later calculations.

\section{Analysis}

The first comparison involved checking the stability of each of the methods. To do this, we integrated across a time change of $1.1 \mathrm{~s}, 2.1 \mathrm{~s}, 3.1 \mathrm{~s}, \ldots, 10.1 \mathrm{~s}$ from two different starting states: the aforementioned initial vector and an increase of $.05 \%$ in all components of the initial vector. We calculated the magnitude of the differences between these two trajectories at each time stop. The results of this process for each of the different algorithms, as well as for the analytical solution, are plotted on the left side of figure 3. The magnitude is given as a percent of the magnitude of the original state vector, i.e. we are examining how the small difference between trajectories increases the Euclidean norm of the state vector over time. This same process was repeated for starting with a decrease in $.05 \%$ of all components in the initial vector and was also compared to starting at the initial vector. These results are shown on the right side of the same figure. All integrations were performed with an absolute tolerance of .0001 and a relative tolerance of $3 \times 10^{-14}$.
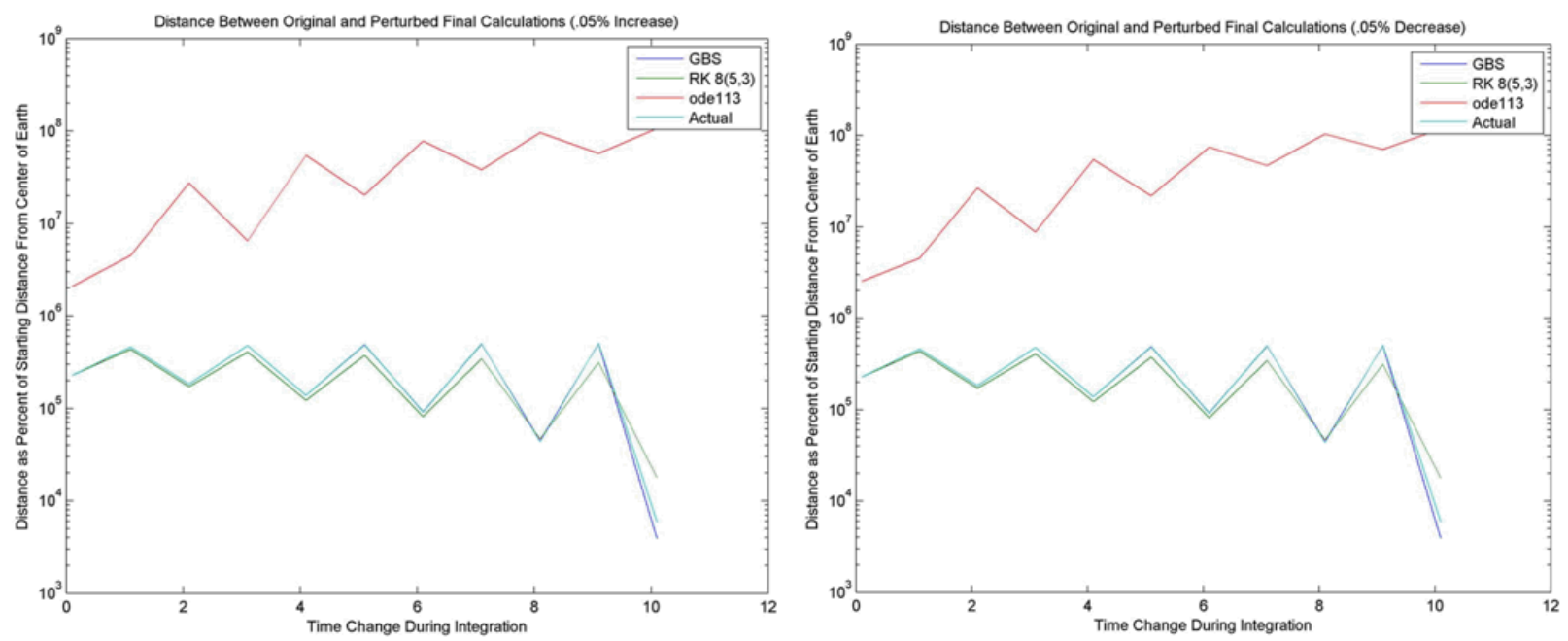

Figure 3-Stability for absolute tolerance of .0001 
As we can see from these plots, a small change is magnified much more quickly by ode113 than for GBS or RK 8(5,3). This means that ode113 is the least stable of the three, most likely because it must begin with a first order estimation before it can increase to higher order estimations. However, none of the methods are necessarily unstable as this analysis was performed with a rather weak error tolerance. In fact, all of this error can be controlled by increasing our level of tolerance as can be seen by the later plots. Therefore, figure 3 only shows that error propagates faster in ode 113 than the other two methods. Thus, these results were further affirmed by repeating these calculations with an absolute error tolerance of $10^{-10}$ and relative error tolerance of $3 \times 10^{-14}$. The results of these calculations are shown in figure 4 .
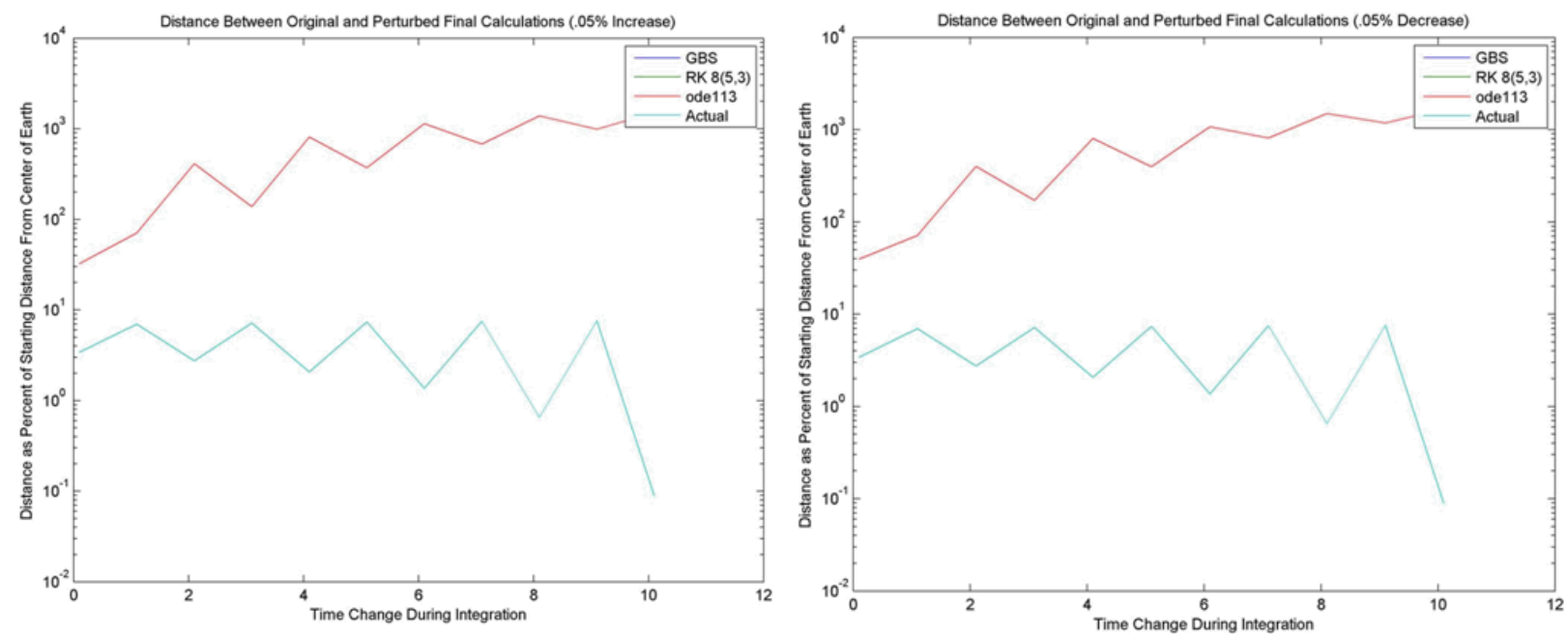

Figure 4-Stability for absolute tolerance of $10^{-10}$

The next point of comparison analyzed was computational efficiency vs. accuracy and precision. This was done by looking at the number of function evaluations vs. absolute error tolerance, computational time vs. absolute error tolerance, function evaluations vs. actual error, and computational time vs. actual error. For this data, each method was integrated over a time change of 500s starting at the initial vector for various levels of absolute error tolerance ranging from $10^{2}$ to $10^{-10}$ and a relative error tolerance of $3 \times 10^{-14}$. The results of the first two plots are shown in figure 5, and the second two are shown in figure 6. 

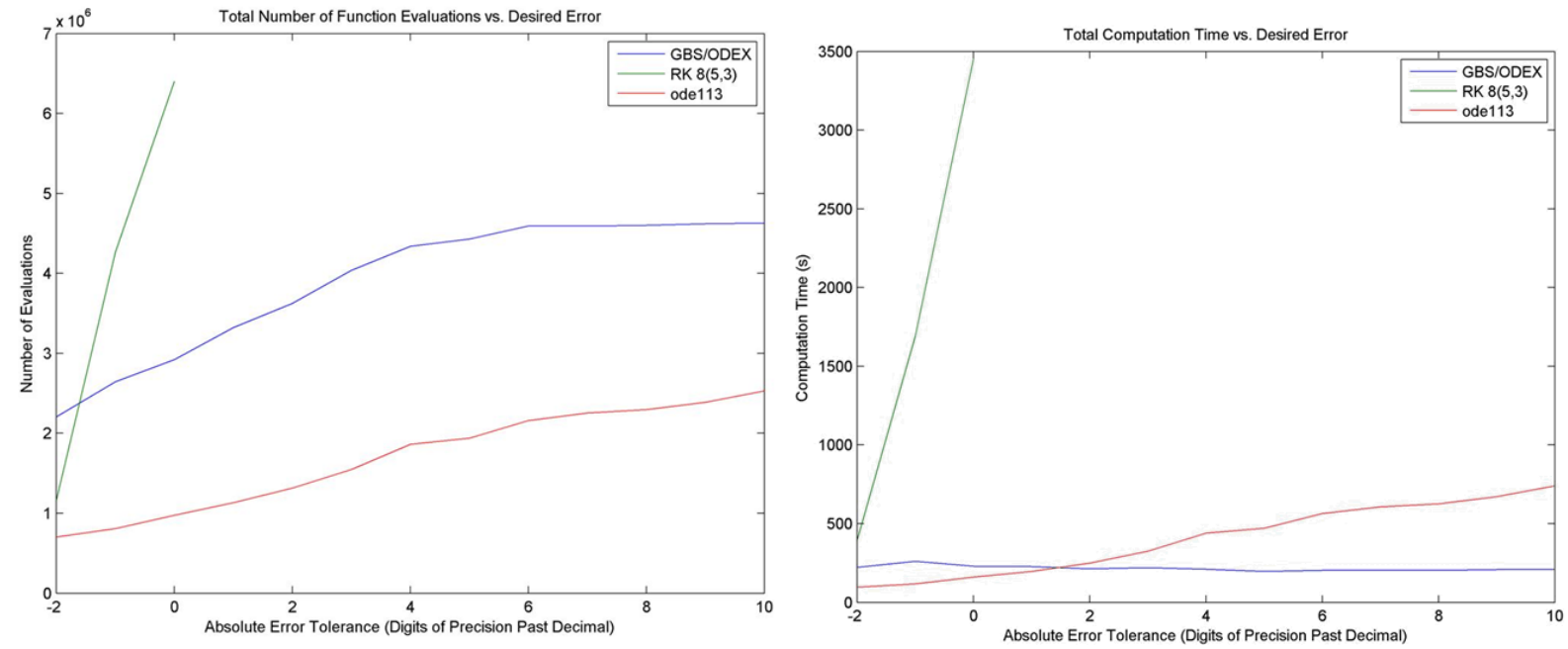

Figure 5-Function evaluations and computational time vs. error tolerance

During evaluation, the RK $8(5,3)$ quickly became much more inefficient than the other two methods to the point that computational time was being restricted by its evaluation. This came even with the effort to optimize the RK $8(5,3)$ factor of safety for stepsize at a relatively low precision of $10^{-4}$. Therefore, its integration was stopped early as its computational time passed the GBS method by a factor of 10. This also suggests that even if the untested higher order Runge-Kutta method 9(8) from [10], [11], or [12] was chosen, it still would be outperformed since only a one order decrease from this level quickly becomes very inefficient.
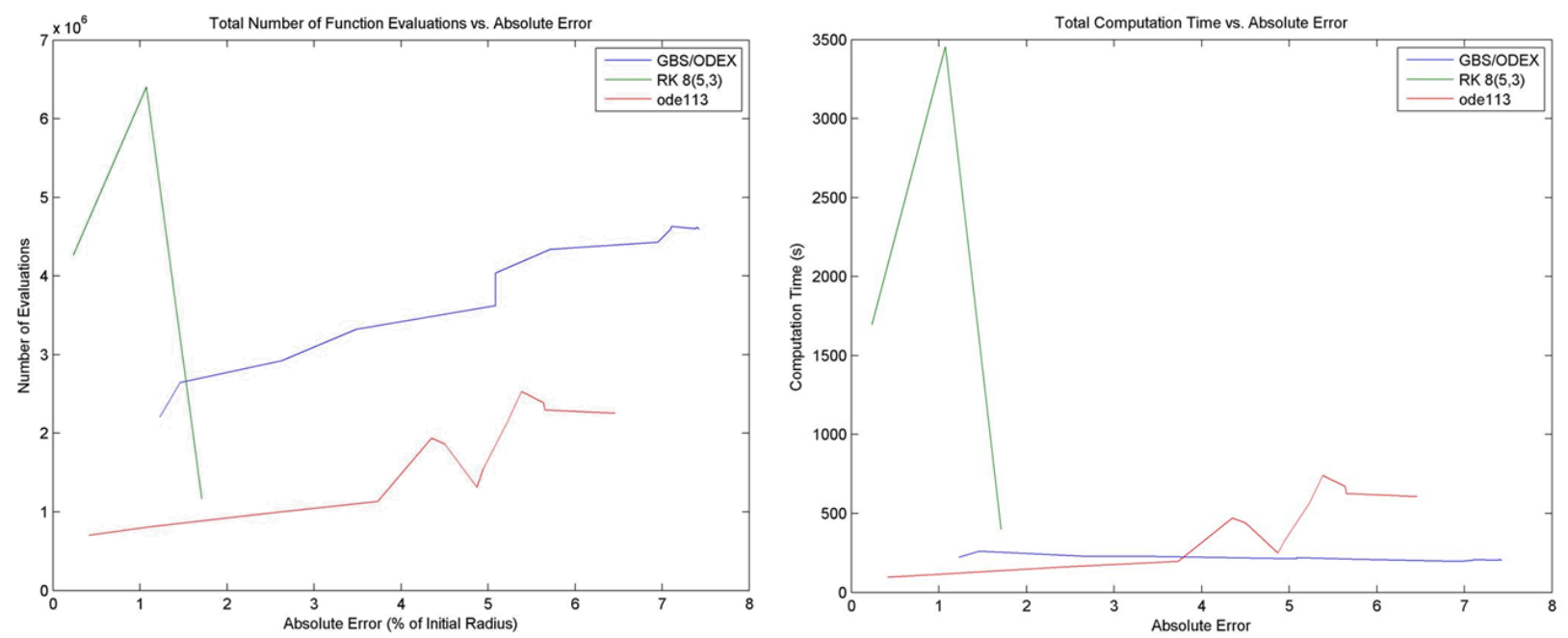

Figure 6-Function evaluations and computational time vs. actual/absolute error

These plots are interesting because they show that even with smaller levels of error tolerance, we may actually increase our actual error. This is not a normal occurrence, though, so if a certain level of error is desired then that should be the error tolerance. In addition, in all cases, the integration still obtains around the desired level of error, but sometimes we get lucky 
and gain some extra precision. However, it does imply the need for stable method as error can be somewhat sporadic.

Consideration of all four plots simultaneously leads to a few conclusions. The most obvious of these is that the RK 8(5,3) method should never be used to integrate for high levels of accuracy/precision on a complicated full-force model as it quickly becomes inefficient. The next is that ode113 seems to require less function evaluations than GBS, but at the same time expends much more time in overhead calculations of new coefficients for the underlying interpolating polynomial at each step. This in turn causes the computational time to increase past that of GBS very quickly. This can be seen by looking at Appendix B, which shows a table of all the data created for the above plotted test runs. In addition, GBS actually becomes more efficient around error tolerances of $10^{-5}$ to $10^{-10}$ while simultaneously decreasing absolute error. This comes as a result of the ability for GBS to utilize incredibly high orders for calculations - in fact orders can sometimes even reach 15 or 20 . This allows GBS to take a much larger stepsize, which keeps it efficient for high precision.

However, the full force model has much more expensive function evaluations, thereby occupying a much larger portion of the computational time. Thus, ode 113 may perform better than GBS at higher precisions under the full force model than it did with the test model. To estimate how much this precision actually is, we first considered the result presented by Montenbruck in [4] that when integrating with a simple model such as our test model or the two body problem, total computing time for extrapolation is 30-60\% higher than expected from just looking at function evaluations, and about 200\% higher for variable order/stepsize multistep methods. By construction of the methods, GBS should always require the same time per step in overhead calculations regardless of function complexity. The same also holds true for ode 113. Combining all of this information leads us to the estimation that one function evaluation of the full force model requires anywhere from $600 \%$ to $1200 \%$ of the time required for one function evaluation of our test model. To account for this, the computational complexity of the test model was multiplied by factors of 10 and 20 (to provide a buffer region in complexity), and then integrated over 100s, beginning from the initial vector. The results are shown in figure 7.
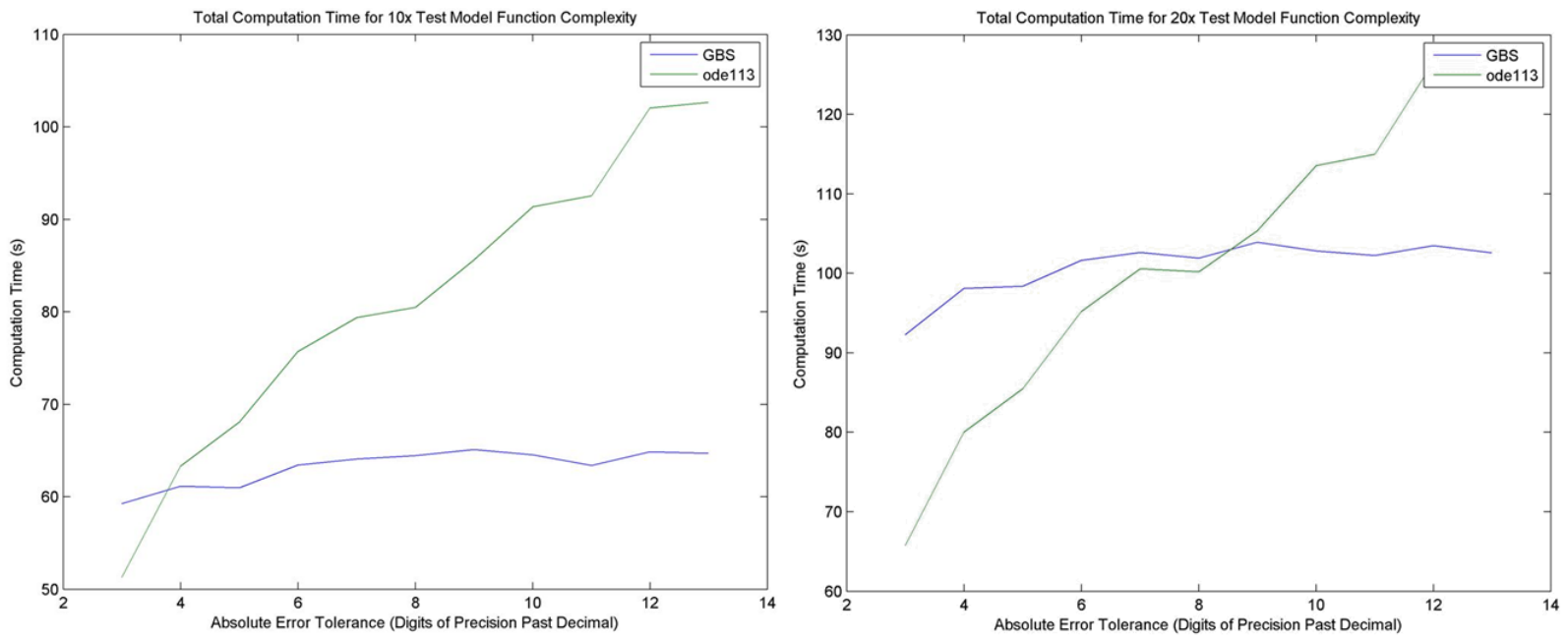

Figure 7-Computation time for increased function complexity 
What we can glean from this figure is that varying levels of computational complexity of the underlying system changes the point at which GBS begins to outperform ode113. As Appendix B shows right now, ode113 only outperforms GBS in computational time through

about $10^{-2}$. However, figure 7 shows that increasing computational complexity by factors of 10 and 20 results in ode113 outperforming GBS through error tolerances of about $10^{-4}$ and $10^{-9}$ respectively.

It is interesting to note, though, that around an absolute error tolerance of $10^{-10}$, the slope of the ode113 curve is steeper than the slope of the GBS curve. Thus, it seems that there should be some level of tolerance past $10^{-10}$ where using GBS may even require less function evaluations than using ode113, making the above analysis of computational time irrelevant at these levels. Thus, GBS will always be the best choice if accuracy and precision are the main concerns. This is consistent with the types of methods, for GBS can create incredibly high order calculations, whereas ode1 13 has a maximum order of 12 , according to documentation found in the ode113 program in MATLAB.

The final question that needed to be considered was whether or not dense output can ever be a better option than straightforward application of the methods. In all practicality, we will only implement dense output interpolation for single-step methods, e.g. Runge-Kutta or extrapolation, for these methods use a much larger stepsize than the multistep methods. This means that the efficiency of the single step methods deteriorates much faster than the efficiency of multistep methods as the number of predefined output points increases. This difference led [4] to conclude that single step methods are inefficient if more than 50 to 100 output points are required in a single revolution. However, in [1] Hairer et. al. completed a thorough error analysis of high order dense interpolation schemes for many different test problems, to include a few in celestial mechanics. Their results indicate that dense interpolation is in fact an efficient, accurate alternative to multistep methods for generating outputs at many different points in a revolution (especially when using their error control schemes for dense output of their GBS extrapolation algorithm). It is important to note, though, that an interpolation scheme will generally have greater error than the underlying method (even though the difference is very small), so error tolerance levels should be adjusted accordingly. We refer the reader to [1] for an investigation on how to adapt to the different error.

\section{Results and Conclusions}

The first main question that needed to be addressed was which method(s) provided the most stability. From the analysis done in this area, we conclude that ode 113 is not as stable as either GBS or RK $8(5,3)$, while these two are about equal in this area. This means that ode113 is more likely to compound errors over time and will be subject to greater global error than either GBS or RK 8(5,3).

With regard to efficiency, we can conclude that RK $8(5,3)$ should not be used as the main integrator at any level of error tolerance. This is because RK $8(5,3)$ quickly becomes very inefficient, even at rather modest levels of error tolerance. On the other hand, the comparison between GBS and ode1 13 is much more interesting. The test evaluations show that for higher accuracy/precision, GBS is definitely better than ode 113 as it affords much smaller error per unit of computational time. However, if modest precision is desired, then ode 113 may in fact outperform GBS. For the 500s test case in this study, GBS is more efficient for error tolerances 
less than or equal to $10^{-2}$, otherwise ode113 is more efficient. As shown in the analysis, this leads us to estimate that, for a full force model with 10 to 20 times as much complexity as the test model, GBS will be more efficient through error tolerances of $10^{-4}$ and $10^{-9}$, respectively. Otherwise, ode113 is more efficient. Results from the actual error for the 500s test cases confirm this conclusion - ode113 exhibited smaller error for absolute tolerances greater than $10^{-2}$ while GBS returned smaller error for tolerances less than or equal to $10^{-2}$. Analogous results were found for the other test cases as well.

To extend these results to actual units in an entire orbit, we can safely assume that error compounds linearly for a given level of tolerance since we control error via the built in error estimators, which adds about the same amount of error at each step. Examining the errors shown in Appendices B and C, we see that the pattern of absolute errors returned by GBS is much more regular than ode113, meaning that smaller error tolerances are more likely to produce smaller actual errors in GBS as opposed to ode113. Using these appendices to relate error tolerance to actual error and taking into consideration the higher stability of GBS, we conclude the following for a 90 min orbit: GBS is more stable and more efficient for absolute errors less than $100 \mathrm{~m}$, GBS and ode 113 are comparable in efficiency for actual errors between $100 \mathrm{~m}$ and $1000 \mathrm{~m}$, and ode113 is more efficient while maintaining sufficient stability for actual errors greater than $1000 \mathrm{~m}$ (these estimates only hold true for this specific test model). This estimate was obtained by using the absolute error in the state vector as an estimate for the distance in meters of the error tolerance, which is valid because the speed portion of the state vector has a negligible impact on the magnitude of the vector, meaning that the magnitude is a close approximation of the error in meters. For larger orbits, a safe estimate is that GBS is a better method for errors less than about $1 \mathrm{~km}$, the two are comparable between $1 \mathrm{~km}$ and $10 \mathrm{~km}$, and ode 113 is better for errors larger than $10 \mathrm{~km}$. Our only reservation about this conclusion is that the test model did not incorporate nonlinear terms because we wanted to incorporate drag and nonautonomous perturbations while maintaining the existence of a closed-form solution. This may have added instability in the methods, while simultaneously distorting the gravity term. However, the test cases in [4] incorporated nonlinear terms and also had an analytical solution which showed that both methods were stable under these situations, so we can reasonably accept our conclusions. In addition, extra distortion of the gravity term tends to exaggerate the effect of perturbations, meaning that our test model should do well at taking perturbations into account.

In practical usage, if we are in the region of error tolerance where we want to use a GBS extrapolation scheme, then we should be considering the possibility of implementing dense output interpolation. Now, dense output creates a different polynomial at each step, meaning that we only want to use it if we have predefined output points to find (extending the results of [4] we recommend dense output for more than 50-100 points per orbit). In the case that we have all of these predefined output points prior to integration, we can simply use the extrapolation method to integrate from the starting time to the time that is furthest away in the list of output points, using dense interpolation to find the points in between. This can be done by running a simple test at each step to see if any of the desired output points lie in that time step. In the case where we do not know the output points prior to integration, but think that we may need to find more than 50-100, we can store points at a set interval of steps (maybe every $10^{\text {th }}$ step) and then integrate from those points. This method is much preferable to the alternative of simply storing final output points as it minimizes the number of integrations necessary while also maintaining an almost set time interval between output points. If memory is a consideration, then dense output can be used to store points at set time intervals (instead of after a set number of steps), and 
then knowledge of these equal time intervals can be used to compress the data. Both of these methods eliminate the need to store only final outputs from integrations which require high memory storage and computation time rather than one or the other.

\section{Recommendation}

When integrating satellite orbits, we recommend using GBS if accuracy and precision is essential, and ode113 if a less accurate estimate is acceptable but efficiency is the largest concern. If a mix of the two is required, then we recommend using GBS for integration.

When we refer to these methods, we specifically suggest using the GBS/ODEX algorithm presented in [1] whenever we recommend using GBS because this is the most advanced model that we saw in literature. Also, when we recommend using the built-in method ode 113 from MATLAB, there are also other comparable variable order/variable stepsize predictor corrector methods that can be implemented (such as DE, see [9] for details on the similar methods to ode113). In fact, ode113 is based on one of these according to [9].

If we are in the region of error tolerance where we want to use GBS extrapolation, and if more than 50-100 output points are known to be needed prior to integration, then we recommend integrating from the starting time to the time that is farthest away and using dense interpolation to find the points in the middle. If we are in this region of error tolerance and we know that we will need to find at least 50-100 predefined output points, but we do not yet know where these points will be located, we recommend implementing one of two methods. The first of these is to store points at a set interval of steps, maybe every $10^{\text {th }}$ step, and then integrate from these stored points. If memory is also a consideration, then we can use dense output to store points at set time intervals, and use this knowledge to compress the data. 


\section{Appendix A-Closed form solution to test model}

$$
\begin{gathered}
x(t)=e^{\alpha t}\left(C_{1} \cos (\beta t)+C_{2} \sin (\beta t)\right)+A_{1} \cos \left(\frac{t}{1000}\right)+B_{1} \sin \left(\frac{t}{1000}\right) \\
y(t)=e^{\alpha t}\left(C_{3} \cos (\beta t)+C_{4} \sin (\beta t)\right)+A_{3} \cos \left(\frac{t}{1000}\right)+B_{4} \sin \left(\frac{t}{1000}\right) \\
z(t)=e^{\alpha t}\left(C_{5} \cos (\beta t)+C_{6} \sin (\beta t)\right)+A_{1} \cos \left(\frac{t}{1000}\right)+B_{1} \sin \left(\frac{t}{1000}\right) \\
v_{x}(t)=e^{\alpha t}\left[C_{1}(\alpha \cos (\beta t)-\beta \sin (\beta t))+C_{2}(\beta \cos (\beta t)+\alpha \sin (\beta t)]-\frac{A_{1}}{1000} \cos \left(\frac{t}{1000}\right)\right. \\
\quad+\frac{B_{1}}{1000} \sin \left(\frac{t}{1000}\right) \\
v_{y}(t)=e^{\alpha t}\left[C_{3}(\alpha \cos (\beta t)-\beta \sin (\beta t))+C_{4}(\beta \cos (\beta t)+\alpha \sin (\beta t)]-\frac{A_{2}}{1000} \cos \left(\frac{t}{1000}\right)\right. \\
\quad+\frac{B_{2}}{1000} \sin \left(\frac{t}{1000}\right) \\
v_{z}(t)=e^{\alpha t}\left[C_{5}(\alpha \cos (\beta t)-\beta \sin (\beta t))+C_{6}(\beta \cos (\beta t)+\alpha \sin (\beta t)]-\frac{A_{1}}{1000} \cos \left(\frac{t}{1000}\right)\right. \\
+\frac{B_{1}}{1000} \sin \left(\frac{t}{1000}\right)
\end{gathered}
$$

$$
\begin{gathered}
\alpha=\operatorname{Re}\left(\frac{-D+\sqrt{\left.D^{2}+4 K\right)}}{2}\right) \\
\beta=\operatorname{Im}\left(\frac{-D+\sqrt{\left.D^{2}+4 K\right)}}{2}\right) \\
A_{1}=\frac{D\left(-5 \times 10^{9}\right)}{D^{2}\left(10^{6}\right)+\left(1+K\left(10^{6}\right)\right)^{2}} \\
B_{1}=\frac{\left(-5 \times 10^{6}\right)\left(1+K\left(10^{6}\right)\right)}{D^{2}\left(10^{6}\right)+\left(1+K\left(10^{6}\right)\right)^{2}} \\
C_{2}=\frac{1}{\beta}\left(v_{x}(0)-C_{1} \alpha-\frac{B_{1}}{1000}\right) \\
C_{6}=\frac{1}{\beta}\left(v_{z}(0)-C_{5} \alpha-\frac{B_{1}}{1000}\right) \\
C_{4}=\frac{1}{\beta}\left(v_{y}(0)-C_{3} \alpha-\frac{B_{2}}{1000}\right) \\
C_{5}=y(0)-A_{2}
\end{gathered}
$$




\section{Appendix B-Data from 500 s run of test model starting from the initial vector}

Statistics for GBS, Not Dense

$\begin{array}{cllllll}\text { atol } & \text { Abs Err } & \text { Comp Time }(s) & \text { Total Evals } & \text { Total Steps } & \text { Rej Steps } & \text { Ave Step Size } \\ 100 & 392060.99 & 223.74 & 2207016 & 63548 & 2 & 0.00787 \\ 10 & 231404.96 & 260.71 & 2646511 & 68581 & 1 & 0.00729 \\ 1 & 15809.662 & 229.78 & 2924750 & 61366 & 30 & 0.00815 \\ 0.1 & 2172.3823 & 228.47 & 3323516 & 59722 & 288 & 0.00841 \\ 0.01 & 54.612532 & 214.33 & 3624922 & 54402 & 1 & 0.00919 \\ 0.001 & 54.461768 & 221.82 & 4038266 & 54532 & 41 & 0.00918 \\ 0.0001 & 12.889268 & 210.94 & 4338899 & 50579 & 1 & 0.00989 \\ 1 \mathrm{e}-005 & 0.74946137 & 196.67 & 4432527 & 46169 & 9 & 0.01083 \\ 1 \mathrm{e}-006 & 0.2505972 & 203.28 & 4592782 & 47296 & 68 & 0.01059 \\ 1 \mathrm{e}-007 & 0.53701521 & 203.89 & 4591073 & 47207 & 126 & 0.01062 \\ 1 \mathrm{e}-008 & 0.27440444 & 204.55 & 4600866 & 47460 & 112 & 0.01056 \\ 1 \mathrm{e}-009 & 0.26235793 & 208.8 & 4621672 & 48184 & 129 & 0.01040 \\ 1 \mathrm{e}-010 & 0.51799214 & 209.19 & 4629045 & 48417 & 126 & 0.01035\end{array}$

Statistics for Runge-Kutta 8(5,3), Not Dense

$\begin{array}{cllllll}\text { atol } & \text { Abs Err } & \text { Comp Time (s) } & \text { Total Evals } & \text { Total Steps } & \text { Rej Steps } & \text { Ave Step Size } \\ 100 & 129280.51 & 396.31 & 1165500 & 97125 & 66 & 0.00515 \\ 10 & 3896644 & 1696.1 & 4269216 & 355768 & 153623 & 0.00247 \\ 1 & 564851.09 & 3454.8 & 6404100 & 533675 & 242580 & 0.00172\end{array}$

Statistics for Shampine-Reichelt ode113, Not Dense

$\begin{array}{cllllll}\text { atol } & \text { Abs Err } & \text { Comp Time }(s) & \text { Total Evals } & \text { Total Steps } & \text { Rej Steps } & \text { Ave Step Size } \\ 100 & 2561189 & 97.732 & 704738 & 352162 & 413 & 0.00142 \\ 10 & 527765.4 & 118.41 & 806544 & 403267 & 9 & 0.00124 \\ 1 & 25789.537 & 159.88 & 976001 & 487982 & 36 & 0.00102 \\ 0.1 & 1234.2579 & 197.98 & 1132490 & 566073 & 343 & 0.00088 \\ 0.01 & 89.291882 & 250.28 & 1313134 & 656566 & 1 & 0.00076 \\ 0.001 & 75.863337 & 325.75 & 1547785 & 773890 & 4 & 0.00065 \\ 0.0001 & 210.65192 & 440.77 & 1863942 & 931970 & 1 & 0.00054 \\ 1 \mathrm{e}-005 & 297.45475 & 471.55 & 1941568 & 970783 & 1 & 0.00052 \\ 1 \mathrm{e}-006 & 39.213414 & 564.16 & 2160334 & 1080107 & 119 & 0.00046 \\ 1 \mathrm{e}-007 & 2.3047878 & 606.68 & 2252519 & 1124297 & 3924 & 0.00045 \\ 1 \mathrm{e}-008 & 14.834154 & 626.14 & 2297166 & 1146656 & 3853 & 0.00044 \\ 1 \mathrm{e}-009 & 15.429796 & 670.52 & 2388832 & 1192610 & 3611 & 0.00042 \\ 1 \mathrm{e}-010 & 27.632078 & 740.13 & 2533691 & 1265473 & 2744 & 0.00040\end{array}$

Note: Abs Err is the Euclidean Norm of the error vector 


\section{Appendix C-Data from 100s run of test model starting from the initial vector with increased function complexities}

Statistics for GBS, 10x Function Complexity

$\begin{array}{lllllll}\text { atol } & \text { Abs Err } & \text { Comp Time } & \text { Total Evals } & \text { Total Steps } & \text { Rej Steps } & \text { Ave Step Size } \\ 0.001 & 12.232453 & 59.25 & 808831 & 10953 & 12 & 0.00914 \\ 0.0001 & 2.8688515 & 61.149 & 866717 & 10091 & 1 & 0.00991 \\ 1 \mathrm{e}-005 & 0.13425077 & 60.974 & 880312 & 8972 & 1 & 0.01115 \\ 1 \mathrm{e}-006 & 0.069040486 & 63.444 & 915522 & 9352 & 13 & 0.01071 \\ 1 \mathrm{e}-007 & 0.085033552 & 64.098 & 924476 & 9622 & 35 & 0.01043 \\ 1 \mathrm{e}-008 & 0.087997512 & 64.44 & 922557 & 9629 & 22 & 0.01041 \\ 1 \mathrm{e}-009 & 0.089856153 & 65.097 & 935510 & 10042 & 35 & 0.00999 \\ 1 \mathrm{e}-010 & 0.086921635 & 64.544 & 928228 & 9784 & 32 & 0.01025 \\ 1 \mathrm{e}-011 & 0.081937314 & 63.414 & 917472 & 9400 & 22 & 0.01066 \\ 1 \mathrm{e}-012 & 0.086592857 & 64.883 & 933486 & 9960 & 35 & 0.01008 \\ 1 \mathrm{e}-013 & 0.07850433 & 64.684 & 915437 & 9335 & 25 & 0.01074\end{array}$

Statistics for ode113, 10x Function Complexity

$\begin{array}{lllllll}\text { atol } & \text { Abs Err } & \text { Comp Time } & \text { Total Evals } & \text { Total Steps } & \text { Rej Steps } & \text { Ave Step Size } \\ 0.001 & 2.7368396 & 51.294 & 313753 & 156875 & 2 & 0.00064 \\ 0.0001 & 0.36105851 & 63.3 & 375362 & 187680 & 1 & 0.00053 \\ 1 \mathrm{e}-005 & 1.2974144 & 68.088 & 398814 & 199406 & 1 & 0.00050 \\ 1 \mathrm{e}-006 & 0.47633763 & 75.687 & 433444 & 216717 & 9 & 0.00046 \\ 1 \mathrm{e}-007 & 0.075809975 & 79.405 & 451488 & 225346 & 795 & 0.00045 \\ 1 \mathrm{e}-008 & 0.10192372 & 80.488 & 456742 & 228004 & 733 & 0.00044 \\ 1 \mathrm{e}-009 & 0.37941862 & 85.598 & 478016 & 238663 & 689 & 0.00042 \\ 1 \mathrm{e}-010 & 0.1125803 & 91.35 & 504250 & 251834 & 581 & 0.00040 \\ 1 \mathrm{e}-011 & 0.17822356 & 92.575 & 512704 & 256103 & 497 & 0.00039 \\ 1 \mathrm{e}-012 & 0.0094157452 & 102.03 & 554248 & 276948 & 351 & 0.00036 \\ 1 \mathrm{e}-013 & 0.43999437 & 102.66 & 550805 & 275157 & 490 & 0.00036\end{array}$

Note: Abs Err is the Euclidean Norm of the error vector 


\section{Appendix C-continued}

Statistics for GBS, 20x Function Complexity

$\begin{array}{lllllll}\text { atol } & \text { Abs Err } & \text { Comp Time } & \text { Total Evals } & \text { Total Steps } & \text { Rej Steps } & \text { Ave Step Size } \\ 0.001 & 12.232453 & 92.287 & 808831 & 10953 & 12 & 0.00914 \\ 0.0001 & 2.8688515 & 98.093 & 866717 & 10091 & 1 & 0.00991 \\ 1 \mathrm{e}-005 & 0.13425077 & 98.387 & 880312 & 8972 & 1 & 0.01115 \\ 1 \mathrm{e}-006 & 0.069040486 & 101.65 & 915522 & 9352 & 13 & 0.01071 \\ 1 \mathrm{e}-007 & 0.085033552 & 102.62 & 924476 & 9622 & 35 & 0.01043 \\ 1 \mathrm{e}-008 & 0.087997512 & 101.9 & 922557 & 9629 & 22 & 0.01041 \\ 1 \mathrm{e}-009 & 0.089856153 & 103.95 & 935510 & 10042 & 35 & 0.00999 \\ 1 \mathrm{e}-010 & 0.086921635 & 102.81 & 928228 & 9784 & 32 & 0.01025 \\ 1 \mathrm{e}-011 & 0.081937314 & 102.27 & 917472 & 9400 & 22 & 0.01066 \\ 1 \mathrm{e}-012 & 0.086592857 & 103.48 & 933486 & 9960 & 35 & 0.01008 \\ 1 \mathrm{e}-013 & 0.07850433 & 102.57 & 915437 & 9335 & 25 & 0.01074\end{array}$

Statistics for ode113, 20x Function Complexity

$\begin{array}{lllllll}\text { atol } & \text { Abs Err } & \text { Comp Time } & \text { Total Evals } & \text { Total Steps } & \text { Rej Steps } & \text { Ave Step Size } \\ 0.001 & 2.7368396 & 65.773 & 313753 & 156875 & 2 & 0.00064 \\ 0.0001 & 0.36105851 & 79.995 & 375362 & 187680 & 1 & 0.00053 \\ 1 \mathrm{e}-005 & 1.2974144 & 85.505 & 398814 & 199406 & 1 & 0.00050 \\ 1 \mathrm{e}-006 & 0.47633763 & 95.19 & 433444 & 216717 & 9 & 0.00046 \\ 1 \mathrm{e}-007 & 0.075809975 & 100.57 & 451488 & 225346 & 795 & 0.00045 \\ 1 \mathrm{e}-008 & 0.10192372 & 100.22 & 456742 & 228004 & 733 & 0.00044 \\ 1 \mathrm{e}-009 & 0.37941862 & 105.37 & 478016 & 238663 & 689 & 0.00042 \\ 1 \mathrm{e}-010 & 0.1125803 & 113.55 & 504250 & 251834 & 581 & 0.00040 \\ 1 \mathrm{e}-011 & 0.17822356 & 114.97 & 512704 & 256103 & 497 & 0.00039 \\ 1 \mathrm{e}-012 & 0.0094157452 & 126.53 & 554248 & 276948 & 351 & 0.00036 \\ 1 \mathrm{e}-013 & 0.43999437 & 126.23 & 550805 & 275157 & 490 & 0.00036\end{array}$

Note: Abs Err is the Euclidean Norm of the error vector 


\section{References}

[1] Hairer, E., Nørsett, S.P., and Wanner, G. 1993, Solving Ordinary Differential Equations I. Nonstiff Problems, $2^{\text {nd }}$ ed. (New York: Springer).

[2] Hairer E. and Ostermann A. 1990, "Dense Output for Extrapolation Methods," Numerische Mathematik, vol. 58, pp. 419-439.

[3] MATLAB Product Support 2010, "Differential Equations in MATLAB," The MathWorks, Inc., at http://www.mathworks.com/support/tech-notes/1500/1510.html\#stiff

[4] Montenbruck, O. 1992, "Numerical Integration Methods for Orbital Motion," Celestial Mechanics and Dynamical Astronomy, vol. 53, pp. 59-69.

[5] Montenbruck, O. and Eberhard, G. 2000, Satellite Orbits, Models, Methods, Applications, (New York, Springer).

[6] Numerical Recipes Software 2007, "Routine Implementing an Eighth-order Runge-Kutta Method," Numerical Recipes Webnote No. 20, at http://www.nr.com/webnotes?20.

[7] Numerical Recipes Software 2007, "StepperBS Implementations," Numerical Recipes Webnote No. 21, at http://www.nr.com/webnotes?21.

[8] Press, W.H., Teukolsky, S.A., Vetterling, W.T., and Flannery, B.P. 2007, Numerical Recipes: The Art of Scientific Computing, $3^{\text {rd }}$ ed. (New York: Cambridge University Press).

[9] Shampine, L.F. and Reichelt, M.W. 1997, “The MATLAB ODE Suite," SIAM Journal on Scientific Computing, vol. 18, no. 1, pp. 1-22.

[10] Sharp, P.W. and Verner, J.H. 1998, “Generation of High-Order Interpolants for Explicit RungeKutta Pairs," ACM Transactions on Mathematical Software, vol. 24, no. 1, pp. 13-29.

[11] Tsitouras, C. 2001, “Optimized Explicit Runge-Kutta Pair of Orders 9(8)," Applied Numerical Mathematics, vol. 38, pp.123-134.

[12] Verner, J.H. 1996, "High-Order Explicit Runge-Kutta Pairs With Low Stage Order," Applied Numerical Mathematics, vol. 22, pp. 345-357. 